(Aus dem physiologischen Institute der Universität Graz.)

\title{
Versuche über die Ermüdbarkeit des markhaltigen Nerven.
}

\author{
Von
}

Dr. Ludwig Haberiandt (Berlin), gew. Assistenten am Institute.

(Hierzu Tafel V:)

Die Lehre von der "Unermüdbarkeit" des Nerven, zu der besonders die Untersuchungen von Bernstein ${ }^{1}$ ), Wedensky ${ }^{2}$ ), Bowditeh ${ }^{3}$ ), Lambert ${ }^{4}$ ), Brodie und Halliburton ${ }^{5}$ ) u. A. geführt hatten, eine Anschauung, die geraume Zeit von den meisten Forschern anerkannt wurde, hat sich im Laufe der letzten Jahre im strengen Sinne des Wortes als nicht haltbar erwiesen. Zwar hatten sich schon in jener Zeit vereinzelte Stimmen des Zweifels erhoben, wie dies unter anderem besonders klar und entschieden in einem Ausspruche Pflüger's ${ }^{6}$ ). zum Ausdruck kommt, den er im Jahre 1891 getan hat:

„Die Ansicht einiger neuerer Forscher von der Unermüdlichkeit der Nerven, die mit allen übrigen guten Tatsachen der Physiologie im Widerspruch steht, wird schon durch die Überlegung, dass die Reizung der Nerven mit Entladung elektrischer Ströme verknüpft

1) Bernstein, Über Ermüdung und Erholung des Nerven. Arch. f. d. ges. Physiol. Bd. 15 S. 289. 1877.

2) Wedensky, Wie rasch ermüdet der Nerv? Zentralbl. f. med. Wissensch. 1884 S. 65.

3) Bowditch, Nachweis der Unermüdbarkeit des Säugetiernerven. Arch. f. Anat. u. Physiol, physiol. Abt. 1890 S. 505 .

4) L a mbert, De l'infatigabilité des nerfs sécrétoires. Compt. rend. de la soc. de Biol. 1894 p. $511-512$.

5) Brodie and Halliburton, Fatigue in non medullared nerves. Journ. of Physiol. vol. 28 p. 181-200.

6) E. P flüg er, Einige Erklärungen, betreffend meinen Aufsatz „Die Quelle der Muskelkraft. Vorläufiger Abriss." Arch. f. d. ges. Physiol. Bd. 50 S. 337. 1891. Pflüger's Archiv für Physiologie. Bd. 137. 
ist, widerlegt. Denn jeder elektrische Strom erzeugt Wärme und bedingt einen Stoffverbrauch. Ebenso ist die tiefe Übereinstimmung, die in den elektrophysiologischen Gesetzen zwischen Muskel und Nerv besteht, ganz unvereinbar mit der Ansicht, dass in der wichtigsten Eigenschaft beide Gewebe sich so grundsätzlich unterscheiden sollten."

Am marklosen Nerven hat zuerst Garten ${ }^{1}$ ), und zwar am Nervus olfactorius des Hechtes eine Ermüdbarkeit nachgewiesen; er reizte den Nerven mit gleichgerichteten Induktionsschlägen rhythmisch in kurzen Intervallen und fand hierbei schon nach wenigen Schwankungen eine Abnahme der Ausschläge des Kapillarelektrometers. Später kam Burian ${ }^{2}$ ) in Untersuchungen am Cephalopodennerven zu analogen Ergebnissen.

Am markhaltigen Nerveu konnte als Erster Fröhlich ${ }^{3}$ ) ein sicheres Ermüdungssymptom aufdecken. Es gelang ihm nämlich, die sogenannte "paradoxe Modifikation der Nervenleitung", wie sie zuerst von $\mathrm{W}$ edensky${ }^{4}$ ) in einem bestimmten Stadium der Narkose beobachtet worden war, auf eine statthabende Ermüdung des Nerven zurückzufülıren, sowie gewisse, ebenfalls schon von Wedensky am Nerven beobachtete Hemmungserscheinungen als Ermüdungssymptome zu erklären. In jüngster Zeit hat endlich im Anschlusse an die Untersuchungen von v. Baeyer ${ }^{5}$ ) und Fröhlich ${ }^{6}$ ) über die Erstickung des Nerven, die für die Frage nach seiner Ermüdbarkeit von grosser Bedeutung wurden, Thörne ${ }^{7}$ ) einen neuen Beweis für

1) Garten, Beiträge zur Physiologie des marklosen Nerven, nach Untersuchungen am Riechnerven des Hechtes. Fis cher, Jena 1903.

2) Burian, Ermüduug und Erholung des Nerven, nach Untersuchungen am Cephalopodennerven. Arch. internat. de Physiol. Vol. 5. 1907.

3) Fr. W. Fröhlich, Ermüdung des markhaltigen Nerven. Zeitschr. f. allgem. Physiol. Bd. 3 S. 468. 1904.

4) Wedensky, Die fundamentalen Eigenschaften des Nerven unter Einwirkung einiger Gifte. Arch. f. d. ges. Physiol. Bd. 82 S. 134.1900. Excitation, inhibition et narcose. (Compt. rend. d. V. Congrès international de Physiol. à Turin.) St. Petersbourg 1901. - Erregung, Hemmung und Narkose. Arch. f. d. ges. Physiol. Bd. 100 S. 1. 1908.

5) v. Ba eyer, Das Sauerstoff bedürfnis des Nerven. Zeitschr. f. allgem. Physiol. Bd. 3 S. 169. 1903.

6) Fr. W. Fröhlich, Das Sauerstoff bedürfnis des Nerven. Zeitschr. f. allgem. Physiol. Bd. 3 S. 131. 1903.

7) W. Thörner, Die Ermüdung des markhaltigen Nerven. Zeitschr. f. allgem. Physiol。 Bd. 7 S. 530. 1908. - Weitere Untersuchungen über die Er- 
dieselbe liefern können, indem er zeigte, dass der in Stickstoff gebrachte Nerv bei andauernder tetanischer Reizung seine Erregbarkeit und Leitfähigkeit bedeutend früher verliert als bei Ruhe. Auch ist es ihm gelungen, durch dauernde tetanische Reizung den Nerv auch in Luft in das Stadium der beginnenden Ermüdung zu versetzen. Im Anschlusse hieran stellte er auch Untersuchungen über die Erholung des ermüdeten Nerven an, auf die an späterer Stelle noch Bezug genommen werden soll.

Durch diese Arbeiten ist demnach der sichere Beweis erbracht worden, dass der periphere Nerv bei seiner Tätigkeit unter bestimmten Umständen einer Ermüdung unterliegt.

In der vorliegenden Abhandlung' soll nun über Versuche betreffend das Verhalten der Fortpflanzungsgeschwindigkeit der Nervenerregung bei länger andauernder Tätigkeit des Nerven berichtet werden.

Bereits Rollett ${ }^{1}$ ) hatte im Jahre 1896 in der Einleitung zu der unten zitierten Arbeit den Gedanken ausgesprochen, dass bei fortgesetzter Tätigkeit des Nerven vielleicht eine Abnahme der Fortpflanzungsgeschwindigkeit der Nervenerregung nachzuweisen wäre, was dann wohl als ein echtes Ermüdungssymptom aufgefasst werden müsste. Auch er gehörte demnach zu jenen Forschern, die an der damals allgemein herrschenden Lehre von der Unermüdbarkeit des Nerven bereits gewisse - und wie es sich bald gezeigt hat, berechtigte Zweifel hegten. Nach den ersten darauf hinzielenden Versuchen wurde er jedoch nach anderer Richtung abgelenkt, ohne dass der angedeutete Weg später von ihm oder von anderer Seite wieder betreten worden wäre; dies ist nun von mir in der vorliegenden Arbeit versucht worden.

Ich verwendete zu meinen Versuchen das seinerzeit von Rollett benutzte Myographion, das eine von ihm angegebene Modifikation

müdung des markhaltigen Nerven: Die Ermüdung in Luft und die „scheinbare Erregbarkeitssteigerung". Zeitschr. f. allgem. Physiol. Bd. 10 S. $29.1909 .-$ Weitere Untersuchungen über die Ermüdung des markhaltigen Nerven: Die Ermüdung und die Erholung unter Ausschluss von Sauerstoff. Zeitschr. f. allgem. Physiol. Bd. 10 S. 351. 1910.

1) A. Rollett, Über die Veränderlichkeit des Zuckungsverlaufes quergestreifter Muskeln bei fortgesetzter periodischer Erregung und bei der Erholung nach derselben. Arch. f. d. ges. Physiol. Bd.64 S. 507. 1896. 
des Marey'schen Myographions darstellt. Der von Rollett ${ }^{1}$ ) gegebenen Beschreibung desselben ist auch eine genaue Analyse der Schreibweise beigefügt. Das Myographion wurde während des Versuches mittels Uhrwerks auf einer eisernen Schienenbahn längs des Zylinders des Myographions mit langsamer Geschwindigkeit vorübergefahren, wodurch eine beträchtliche Anzahl von Zuckungskurven zur Anschreibung gelangen konnte.

Als Stromquelle diente ein Akkumulator, der im Laufe der Versuche öfters auf seine Intaktheit geprüft und bei Bedarf gegen einen neugeladenen ausgewechselt wurde. Die Schliessung und Öffnung des primären Stromes wurde in stets genau gleichen Zeitintervallen durch einen automatischen Quecksilberschlüssel besorgt, der durch den rotierenden Zylinder des Myographions in Tätigkeit versetzt wurde, so dass bei jeder Umdrehung der Trommel der Strom einmal geschlossen und geöffnet wurde. Die gleichmässige Rotation der Trommel wurde mit Hilfe eines Foucault'schen Regulators in befriedigender Weise erzielt, wie die mit einer König'schen Hundertschwingungs - Unterbrechungsstimmgabel ausgeführte Kontrolle ergeben hat, wovon noch des näheren bei Besprechung der zeitlichen Auswertung die Rede sein soll. Zwischen der sekundären Spirale des verwendeten grossen Du Bois-Reymond'schen Schlitteninduktoriums und den beiden Elektrodenpaaren befand sich ein rotierender Quecksilberumschalter, der mittels Zahnradübertragung ebenfalls durch die rotierende Trommel des Myographions in Bewegung gesetzt wurde. Derselbe war derart eingerichtet, dass nur die Schliessungsindultionsschläge in das Präparat gelangen konnten, so zwar, dass sie abwechselnd durch das untere und obere Elektrodenpaar dem Nerven in aufsteigender Richtung zugeführt wurden. Der Elektrodenträger bestand aus einer $7 \mathrm{~cm}$ langen Ebonitplatte, als Elektroden dienten $0,5 \mathrm{~mm}$ dicke Platindrähte in $1 \mathrm{~mm}$ Entfernung, die Elektrodenzwischenstrecke betrug $47 \mathrm{~mm}$; das periphere Elektrodenpaar befand sich dabei knapp an dem einen Ende des Trägers. - Der Schreibhebel des Myographions, der aus einem leichten, mit einer feinen Stablspitze versehenen Strohhalm bestand, war $148 \mathrm{~mm}$ lang, während der Muskel und die Belastung mittels Fadens an ihm in einer Entfernung von

1) A. Rollett, Beiträge zur Physiologie der Muskeln. Denkschr. d. kais. Akad. d. Wissensch. in Wien, mathem.-naturwissensch. Klasse Bd. 58 S. 231. 1887. 
$22 \mathrm{~mm}$ von seiner Drehungsachse angriffen. Die Zuckungskurven wurden demnach in einer 6,727 maligen Vergrösserung anfgezeichnet. Vor Beginn jedes Versuches wurde der Schreibhebel über einer unter ihm befindlichen Kreiseinteilung derart eingestellt, dass er sich in seiner Ruhelage senkrecht zur Zylinderachse befand.

Als Versuchstiere dienten im Frühjahre und Sommer eingefangene Wasserfrösche ( $R$. esculenta aus Ungarn) von meist beträchtlicher Grösse. Nach Tötung der Tiere durch Durchschneidung der Wirbelsäule oberhalb des Steissbeines und Ausbohrung von Rückenmark und Gehirn wurden zunächst in gewöhnlicher Weise Nervmuskelpräparate des M. gastrocnemius mit dem N. ischiadicus hergestellt, wobei der Nerv mit grösstmöglichster Schonung und Vorsicht bis zum durchschnittenen Plexus hinauf freipräpariert und daselbst abgetrennt wurde. War sodann das Präparat auf dem Myographion sicher fixiert und der Nerv über die Elektroden gebrückt worden, so wurde zunächst bei fast stillstehender Trommel eine Zuckung angeschrieben, um den Moment der Reizung an jener zu bezeichnen; von dieser Marke aus wurde sodann eine Linie über die ganze Länge der Trommel hin durch Vorüberschieben des Myographions gezeichnet, die als Ausgangspunkt für die Bestimmung der Länge der Latenzstadien bei der Ausmessung der Kurven benutzt wurde. Nachdem hierauf das Myographion bei emporgestelltem Schreibhebel wieder auf seinen ursprünglichen Ausgangsort zurückgeführt worden war, konnte der eigentliche Versuch beginnen, der darin bestand, dass durch abwechslungsweise Nervenreizung von der unteren und oberen Reizstelle eine kontinuierliche längere Reihe von Myogrammpaaren zur Aufzeichnung gelangte, wie je ein solches Paar bekanntlich zuerst Helmholtz zur Bestimmung der Fortpflanzungsgeschwindigkeit im motorischen Kaltblüternerven aufgezeichnet hatte.

Entsprechend der hier benutzten Versuchsanordnung erfolgte, wie aus dem schon früher Erwähnten hervorgeht, bei jeder Trommelumdrehung nach stets gleich grossen Zeitpausen eine Muskelzuckung, abwechselnd von der distalen und proximalen Reizstelle des Nerven ausgelöst, so dass sämtliche Zuckungskurven genau übereinander angeschrieben wurden. Die untere Reizstelle war 5-10 $\mathrm{mm}$ vom Muskel entfernt, die Entfernung der oberen Reizstelle vom Muskel fiel je nach der Grösse des Tieres verschieden weit aus und schwankte zwischen $52-65 \mathrm{~mm}$. Betrug sie mehr als 52 bzw. $57 \mathrm{~mm}$, so 
war der Nerv zwischen den beiden Elektrodenpaaren in leichten Windungen auf dem Elektrodenträger gelagert; die Längenmessung erfolgte dann nach dem Versuche am gerade ausgestreckten Nerven. Der Abstand seiner Durchtrennungsstelle von der oberen Reizstelle betrug in den verschiedenen Versuchen je nach der Länge des Nerven $5-20 \mathrm{~mm}$, meist $15 \mathrm{~mm}$. Während der Versuche waren die Nerven durch eine deckelförmig über den Elektrodenträger gelegte feuchte Kammer vor Vertrocknung sicher geschützt, das kleine Stück derselben ausserhalb jener nahe am Muskel wurde mit einem Wattebäuschchen leicht üherdeckt, das mit physiologischer Kochsalzlösung durchtränkt war.

Was die Grösse der bei diesen isotonischen Versuchen in Anwendung gebrachten Belastung betrifft, so belief sich dieselbe je nach der Grösse des Muskels auf $30-60 \mathrm{~g}$, und zwar wurde bei mittelgrossen Tieren die Belastung mit $30 \mathrm{~g}$, bei grossen Individuen mit $50 \mathrm{~g}$ und bei einigen sehr grossen Versuchstieren mit $60 \mathrm{~g}$ bemessen (dazu das aus Aluminiumblech verfertigte Gewichtsschälchen samt den Aufbängfäden mit ungefähr $1 \mathrm{~g}$ ).

Vor der Besprechung dieser Versuche soll zunächst das Ergebnis der Vorversuche mitgeteilt werden, die bezüglich der Stärke der dabei in Betracht kommenden Stromschleifen ausgeführt wurden. $\mathrm{Zu}$ diesen Versuchen wurde der Nerv eines frisch hergestellten Präparates zunächst an einer Stelle unterbunden und hierauf derart auf das eine der beiden Elektrodenpaare gelagert, dass die ligierte Stelle sich in einer Entfernung von $1-2 \mathrm{~mm}$ muskelwärts davon befand. Sodann wurde jene Reizstärke gesucht, bei welcher bei Schliessung des Primärstromes eben die erste Muskelzuckung zu beobachten war, also die Stromschleifen die Ligaturstelle übersprangen. Diese Prüfung wurde an mebreren Stellen des Nerven vorgenommen und ergab, dass bei Einwirkung der in den Hauptversuchen verwendeten Schliessungsinduktionsschläge im Durchschnitt bei einem Rollenabstand von $120 \mathrm{~mm}$ (ohne Eisenkern) die erste Zuckung auftrat. Bei den Hauptversuchen wurden Rollenabstände von $150-200 \mathrm{~mm}$, meist von $180 \mathrm{~mm}$ (ebenfalls ohne Eisenkern) gewählt, so dass also bei der Bestimmung der Fortpflanzungsgeschwindigkeit ein nennenswerter Einfluss von Stromschleifen wohl kaum statthaben konnte.

Zur A usmessung der erhaltenen Kurven benutzte ich einen grossen Kurvenanalysator von Runne in Heidelberg, welcher 
eine Messung der Kurven unter Lupenvergrösserung mittels Fadenkreuzes bis auf $0,05 \mathrm{~mm}$ erlaubt.

Um recht feine Kurven zu gewinnen, wurde das Glanzpapier, das zum Überziehen der Trommel verwandt wurde, meist nur schwach in der Kampherflamme berusst und andererseits darauf geachtet, dass die Spitze des Schreibhebels so leicht als möglich auf der Schreibfläche gleite; so wurden meistens Kurven gezeichnet, deren Dicke nur $0,05 \mathrm{~mm}$ odex auch noch weniger betrug.

Eine genaue Bestimmung der Umdrehungsgeschwindigkeit der Trommel ergab eine Zeitdauer von 1,4782 Sekunden für eine Umdrehung, wobei die damit zurückgelegte Wegstrecke $420,5 \mathrm{~mm}$ betrug. Letzterer Wert wurde bei der Messung am abgenommenen, fixierten und trockenen Papiere erhalten. Leider liess die Konstruktion des verwendeten Apparates eine grössere Umdrehungsgeschwindigkeit nicht $\mathrm{zu}$, die an und für sich wohl sehr wünschenswert gewesen wäre. Andererseits aber hätte man speziell bei diesen Versuchen die Rotationsgeschwindigkeit kaum noch nennenswert steigern dürfen, da sonst dem bereits mehr oder weniger ermüdeten Muskel nicht die nötige Zeit zu Gebote gestanden wäre, vor Beginn der nächsten Zuckung seine vollkommene Wiedèverlängerung zu erlangen. Wenn demnach die in Betracht kommenden Differenzwerte recht gering ausfielen, so waren sie doch in deutlichem Maasse und in konstanter Weise vorhanden, wie dies unten mitgeteilt werden soll.

Es ergibt sich aus den angeführten Zahlenwerten, dass in diesen Versuchen der Zeitdauer von 0,01 Sekunde auf der Schreibfläche eine Wegstrecke von 2,84 mm entspricht, somit eine Wegstrecke von $1 \mathrm{~mm}$ einer Zeitdauer von 0,0035 Sekunde $(3,5 \sigma)$ gleichzusetzen ist.

Damit stimmt in befriedigender Weise das Ergebnis überein, zu welchem der schon früher erwähnte Kontrollversuch mit einer König'schen Hundertsehwingungs-Unterbrechungsstimmgabel geführt hat.

Bei demselben wurden in 100 Einzelmessungen, die in der für die Hauptversuche allein in Betracht kommenden Trommelgegend der Reizmarke vorgenommen wurden, als Entfernung zweier benachbarter Gipfelpunkte in 76 Fällen eine Distanz von $2,85 \mathrm{~mm}$, in den übrigen 24 Fällen eine Distanz von $2,8 \mathrm{~mm}$ gefunden; daraus berechnet sich der arithmetische Mittelwert von 2,84, genauer von 
2,838 mm. Die damit gleichzeitig ausgeführte Kontrolle bezüglich der Gleichförmigkeit der Umdrehungsgeschwindigkeit liess dieselbe demnach als eine ziemlich weitgehende erscheinen. Der in dieser Hinsicht in Rechnung zu ziehende mittlere Fehler, der sich also auf die geringen Änderungen in der Umdrehungsgeschwindigkeit bezieht, beläuft sich, nach der Methode der kleinsten Quadrate berechnet, auf:

$$
m \cdot F=\sqrt{\frac{0,01^{2} \cdot 76+0,04^{2} \cdot 24}{100}}= \pm 0,021 \mathrm{~mm} .
$$

Derselbe dürfte wohl den Fehlerwert nicht übersteigen, der durch die Ausmessung gegeben erscheint.

Was die Technik dieser betrifft, so wurde die Länge der Latenzstadien stets von dem dem Kurvenanstiege zugekehrten Rande jener vor dem Versuche gezogenen Linie gemessen, welche als Marke für den Reizmoment verwendet wurde. Es sei übrigens dazu bemerkt, dass es sich ja hier nicht um eine möglichst exakte Bestimmung der absoluten Latenzzeiten handelt, sondern es für diese Untersuchungen allein auf die Differenzen derselben ankommt. Der Beginn des Anstieges der Kurven von der Abczisse konnte mittels des benutzten Kurvenanalysators recht genau bestimmt werden; das schief gestellte Fadenkreuz der Lupe wurde stets auf den unteren - im umgekebrten Bilde durch die Lupe gesehen also auf den oberen - Rand der Kurve eingestellt, so dass der Moment des Anstieges derselben bei einiger Übung recht präzis zu bestimmen war. Natürlich wurden zur Ausmessung nur gut gelungene, vor allem genügend fein und gleichmässig angeschriebene Kurven verwertet; bei der grossen Schaar von Kurvenpaaren stand ja vom Anfang und Ende der Zuckungsreihe zur Auswahl für die Messung je eine grössere Anzahl von Kurven zur Verfügung. Unter anderem kam es gelegentlich vor, dass der sehr leichte Schreibhebel durch geringfügige Erschütterungen in kleine Schwankungen geriet, so dass er eventuell vor Beginn der Zuckung die Abszisse nicht ganz gerade anschrieb, sondern dieselbe leichte Senkungen und Erhebungen aufwies, wodurch natürlich die genaue Bestimmung des beginnenden Kurvenanstieges unmöglich gemacht wurde.

Bei den hier zu besprechenden Versuchen lag nun, wie vorauszusehen war, eine beträchtliche Schwierigkeit vor allem darin, dass die einzelnen Zuckungen, die abwechselnd von der unteren und oberen Reizstelle des Nerven ausgelöst wurden, so 
kongruent als nur möglich ausfallen mussten. Dies ist bekanntlich an und für sich nicht immer ohne weiteres zu erreichen, was sich natürlich bei längeren Zuckungsreihen, um die es sich hier handelt, um so mehr bemerklich macht.

War diese wichtige Bedingung für die Vergleichung der Kurven je eines Paares nicht in befriedigender Weise erfullt, was sich oft erst im Verlaufe des Versuches an der Zuekungsreibe durch ungleiche Zuckungshöhen allmählich zeigte, so wurde der Versuch entweder abgebrochen oder später nicht zu genauen Ausmessungen verwertet. Andererseits kam es auch nicht allzu selten vor, dass gerade im Beginn des Versuches die ersten Kurvenpaare nicht ganz regelmässig ausfielen, während sich dann der weitere Verlauf des Versuches oft sehr gleichmässig gestaltete. So war dies z. B. auch bei den ersten zwei Myogrammpaaren des Versuches der Fall, von welchem die reproduzierte Kurvenreihe stammt, die an späterer Stelle eingehender besprochen werden soll. (S. Tafel V und S. 446). Weil solche Unregelmässigkeiten gerade an den allerersten Zuckungen öfters auftraten, wurden bei vielen meiner Versuche diese überhaupt nicht angeschrieben und bei der Zählung der Einzelkurven dann auch nicht berücksichtigt. Endlich sei noch erwähnt, dass bei der genauen Ausmessung wohl in den meisten Fällen ganz geringe, unvermeidliche Differenzen in den Zuckungshöhen an den einzelnen, zusammengehörigen Kurvenpaaren gefunden wurden, die jedoch auf die Differenzwerte der Latenzstadien keinen merklichen Einfluss ausübten.

Die zahlreichen in dieser Art ausgeführten Versuche mit längeren, kontinuierlichen Kurvenreihen von 180-320 Einzelzuckungen haben nun in den meisten Fällen ergeben, dass sich die Differenz der Latenzstadien am Ende einer solchen Zuckungsreihe bei der Ausmessung merklich grösser erweist als zu Beginn des Versuches, was also einer Verzögerung der Fortpflanzungsgeschwindigkeit der Erregung im Nerven während eines derartigen Versuches entsprechen würde. Die Differenz der Latenzzeiten zeigte sich am Ende des Versuches meist um ein Drittel des Anfangswertes vergrössert, bisweilen auch um etwas mehr. Dass diese Verzögerung der Nervenleitung tatsächlich durch die fortgesetzt wiederholte Leitungstätigkeit verursacht sein dürfte, also als ein direktes Ermüdungssymptom aufzufassen wäre, ergibt sich wohl aus dem Umstande, dass eine kurze Pause am 
Schlusse des Versuches genügte, um jenengrösseren Differenzwert auf seinen Anfangswert zurückzubringen. Damit ist jedenfalls ausgeschlosseu, dass diese Zunahme, woran möglicherweise gedacht werden könnte, eine sich im Verlaufe des Versuches entwickelnde Absterbeerscheinung vorstelle, die natürlich als solehe durch Einschaltung einer kurzen Ruhepause nicht rückgängig gemacht werden könnte. Über die Länge der zur Wiederherstellung der ursprünglichen Differenzwerte nötigen Pausen wird später noch berichtet werden. Zunächst möge hier als Beleg für das eben Mitgeteilte ein Beispiel aus den Tabellen des Versuchsprotokolles angeführt werden.

Bei dem Versuche (Nr. 62 vom 11. Juli 1910) wurde ein besonders grosses und äusserst kräftiges, gut genährtes Tier verwendet, das erst kurze Zeit vor der Versendung eingefangen worden war. Da sich der Muskel als sehr leistungsfähig erwies - sämtliche Versuche wurden, wie bereits erwähnt, am entbluteten Muskel gewöhnlicher Nervmuskelpräparate angestellt - konnte eine verhältnismässig recht grosse Zahl von Zuckungen angeschrieben werden, ohne dass der Muskel allzusehr ermüdete. So wurden in diesem Versuche 320 Zuckungen ausgeführt, wobei stets nach je 20 angesehriebenen Zuckungskurven 40 Zuckungen bei abgehobenem Schreibhebel erfolgten; nach der 320. Zuckung wurde sodann eine Pause von einer Minute eingeschaltet, worauf noch weitere 20 Zuckungen registriert wurden.

In der $\mathrm{n}$ achfolge $\mathrm{n}$ d e $\mathrm{n} \mathrm{T}$ a be ll e sind für die einzelnen Latenzstadien und ihre Differenzwerte sowohl die ausgemessenen Streckenlängen als auch die daraus berechneten Zeitgrössen verzeichnet. Sodann folgen die daraus sich ergebenden Werte für die Fortpflanzungsgeschwindigkeit, während in der letzten Kolonne als Zuckungshöhen die an den Kurven direkt gemessenen, nicht reduzierten Ordinaten der Gipfelpunkte vermerkt stehen.

\section{Versuch Nr. 62 am 11. Juli 1910.}

Sehr grosse Rana esculenta mit äusserst kräftiger Muskulatur. Nervmuskelpräparat der rechten Seite. Abstand der Reizstellen vom Muskel $10 \mathrm{bzw}$. $65 \mathrm{~mm}$. Durchtrenuungsstelle $15 \mathrm{~mm}$ von der oberen Reizstelle entfernt. Belastung $60 \mathrm{~g}, \mathrm{R}_{\text {.-A. }} 180 \mathrm{~mm}$. Zimmertemperatur $19^{\circ} \mathrm{C}$. 


\begin{tabular}{|c|c|c|c|c|c|c|c|c|c|}
\hline \multirow{2}{*}{$\begin{array}{l}\text { Zahl der } \\
\text { Zuckungen }\end{array}$} & \multicolumn{4}{|c|}{ atenzstadium als } & \multicolumn{2}{|c|}{$\begin{array}{l}\text { Die Differenz- } \\
\text { werte in }\end{array}$} & \multirow{2}{*}{$\begin{array}{c}\text { Fortpflan- } \\
\text { zungs- } \\
\text { geschwin- } \\
\text { digkeit } \\
\text { pro Sek. } \\
\text { m }\end{array}$} & \multirow{2}{*}{\multicolumn{2}{|c|}{$\begin{array}{l}\text { Zuckungs- } \\
\text { höhen } \\
\text { nicht redu- } \\
\text { ziert } \\
\text { mm }\end{array}$}} \\
\hline & \multicolumn{2}{|c|}{$\begin{array}{c}\text { Wegstrecke } \\
\text { mm }\end{array}$} & \multicolumn{2}{|c|}{$\begin{array}{c}\text { Zeitdauer } \\
\sigma\end{array}$} & $\mathrm{m}$ & $\sigma$ & & & \\
\hline $\begin{array}{rlr}\text { 7. } & \text { und } & 8 . \\
9 . & \text { " } & 10 . \\
11 . & \text { " } & 12 . \\
13 . & \text { " } & 14 . \\
121 . & \text { " } & 122 . \\
181 . & \text { " } & 182 . \\
251 . & \text { " } & 252 . \\
307 . & \text { " } & 308 .\end{array}$ & & & & 8 & & 1,05 & & & 28 \\
\hline $\begin{array}{lll}311 . & \text { " } 312 . \\
15 . & " 316 . \\
119 . & " & 320 .\end{array}$ & & & & & & & & & $\begin{array}{l}21,7 \\
21,1\end{array}$ \\
\hline $\begin{array}{l}\text { 27. " } 328 . \\
37 . ~ " 338 .\end{array}$ & $\begin{array}{l}\mathbf{3 , 4 5} \\
3,70\end{array}$ & 4,00 & 12,95 & 14,00 & 0,3 & 1,05 & $\begin{array}{l}52,38 \\
52,38\end{array}$ & 18,55 & 18,3 \\
\hline
\end{tabular}

Wie schon aus dieser Tabelle hervorgeht, ergab sich also bei der genauen Ausmessung im Verlaufe einer derartigen längeren Zuckungsreihe eine Verlängerung der Differenz der Latenzzeiten, die ein Drittel ihres Anfangswertes beträgt, was demnach eine entsprechende Abnahme der Fortpflanzungsgeschwindigkeit der Nervenerregung (von rund 52 auf rund $39 \mathrm{~m}$ pro Sekunde) bedeuten würde. Zu ganz analogen Ergebnissen führte in gleicher Weise eine beträchtliche Anzahl anderer Versuche, wobei manchmal die Zunahme der Differenzwerte der Latenzstadien eher noch grösser gefunden wurde. Der verhältnismässig hohe Anfangswert für die Fortpflanzungsgeschwindigkeit im Nerven, wie er bei den meisten Versuchen in ziemlich konstanter Weise gefunden wurde, dürfte vielleicht, abgesehen von der höheren Temperatur, in dem Umstande seine Erklärung finden, dass bei diesen Versuchen stets übermaximale und zwar meist annähernd gleich starke Reize in Verwendung kamen. Dass aber bei Einwirkung solcher die Leitungsgeschwindigkeit eine wenn auch nicht gesetzmässige Zunahme erfährt, ist wohl zum mindesten nicht ganz ausgeschlossen, wofür u. a. besonders die Untersuchungen Durig's ${ }^{1}$ ) zu sprechen scheinen, die speziell mit den früheren Beobachtungen von von Vintschga ${ }^{2}$ ) vollkommen übereinstimmen.

1) A. Durig, Wassergehalt und Organfunktion. III. Mitteilung. Arch. f. d. ges. Physiol. Bd. 92 S. 293.1902.

2) M. v. Vintschga u, Untersuchungen über die Frage, ob die Geschwindigkeit der Fortpflanzung der Nervenerregung von der Reizstärke abhängig ist. Arch. f. d. ges. Physiol. Bd. 30 s. 17. 1883. 
Dass ferner die Zunahme der Latenzdifferenzen bei dem Versuche, auf den sich obige Tabelle bezieht, verhältnismässig beträchtlich später zu konstatieren war als bei den meisten früheren Versuchen, kann vielleicht damit zusammenhängen, dass das zu diesem Versuche benutzte Tier aus einer Sendung stammte, welche aus ganz frisch eingefangenen, äusserst gut genährten und kräftigen Tieren bestand.

Die Ruhepause, nach welcher die entsprechenden Differenzwerte wieder ihren Anfangswert erreicht hatten, betrug bei dem angefübrten Versuche eine Minute. Um zu erfahren, welche Zeitdauer eben noch in dieser Beziehung gentugt, wurden Versuche ausgeführt, bei denen die Erholungspause auf 30 und später auf 15 Sekunden abgekürzt wurde. Es zeigte sich hierbei, dass auch schon die kurze Zeit von 15 Sekunden ausreicht; noch kürzere Pausen kamen in den Versuchen nicht zur Anwendung. Dieser Befund wäre wohl mit der sehr interessanten Tatsache in Analogie zu setzen, die, wie bereits einleitend kurz erwähnt wurde, Thörner ${ }^{1}$ ) in seinen Untersuchungen ganz anderer Art aufgefunden hat, dass nämlich sogar die anaërobe Erholung des ermüdeten Nerven bereits nach 10 Sekunden deutlich ausgeprägt ist.

Eine solche kurze Pause von nur 15 Sekunden fand auch am Schlusse jenes Versuches statt, von welchem die reproduzierten Kurven erhalten worden waren (siehe Tafel V). Dabei war das Nervmuskelpräparat der linken Seite eines ebenfalls sehr grossen und kräftigen, frisch eingefangenen Tieres verwendet worden. Die untere Reizstelle war $5 \mathrm{~mm}$, die obere $65 \mathrm{~mm}$ vom Muskel entfernt; der Abstand der Durchtrennungsstelle von der oberen Reizstelle betrug $15 \mathrm{~mm}$.

Der sehr grosse Muskel war mit $60 \mathrm{~g}$ belastet, der Rollenabstand betrug $170 \mathrm{~mm}$. Der grössere Zwischenraum im oberen Teile der Kurvenreibe entspricht 50 nicht angeschriebenen Zuckungen, während nach den nächsten 20 wieder registrierten Zuckungen, zwischen der 224. und 225. Zuckung, die Pause von 15 Sekunden eingeschaltet wurde, was durch die stärkere, horizontale Linie angezeichnet ist.

1) W. Thörner, Weitere Untersuchungen über die Ermüdung des markbaltigen Nerven: Die Ermüdung und die Erholung unter Ausschluss von Sauerstoff. Zeitschr. f. allgem. Physiol. Bd. 10 S. 351. 1910. 
Die Ausmessung führte zu einem analogen Ergebnis wie bei den meisten anderen derartigen Versuchen. Die Zunahme der Differenz der Latenzzeiten betrug hier ebenfalls ein Drittel des Anfangswertes, zeigte sich aber bereits von der 211. Zuckung an ausgebildet. Nach der Pause von 15 Sekunden war die Differenzgrösse wieder auf ihren anfänglichen Wert zurückgegangen. Es sei noch erwähnt, dass nach 100 Zuckungen die Differenz der Latenzzeiten in Vergleich zum Beginn des Versuchs noch nicht merklich verändert gefunden wurde, während die Messung im Beginn der zweiten Hälfte des zweiten Hunderts bereits eine geringfügige Vergrösserung der Differenzen nachweisen konnte, die aber erst im dritten Hundert ein Drittel des Anfangswertes erreichte. Aus der nachstehenden Tabelle sind die angeführten Einzelheiten übersichtlich und zahlenmässig zu entnehmen.

\section{Versuch Nr. 67 am 14. Juli 1910.}

Zimmertemperatur $19^{\circ} \mathrm{C}$.

\begin{tabular}{|c|c|c|c|c|c|c|c|c|c|}
\hline \multirow{2}{*}{$\begin{array}{l}\text { Zahl der } \\
\text { Zuckungen }\end{array}$} & \multicolumn{4}{|c|}{ Latenzstadium als } & \multicolumn{2}{|c|}{$\begin{array}{c}\text { Die Differenz- } \\
\text { werte in }\end{array}$} & \multirow{2}{*}{$\begin{array}{c}\text { Fortpflan- } \\
\text { zungs- } \\
\text { geschwin- } \\
\text { digkeit } \\
\text { pro Sek. } \\
\text { m }\end{array}$} & \multirow{2}{*}{\multicolumn{2}{|c|}{$\begin{array}{l}\text { Zuckungs- } \\
\text { höhen } \\
\text { nicht redu- } \\
\text { ziert } \\
\text { mm }\end{array}$}} \\
\hline & \multicolumn{2}{|c|}{$\begin{array}{l}\text { Wegstrecke } \\
\text { mm }\end{array}$} & \multicolumn{2}{|c|}{$\begin{array}{l}\text { Zeitdauer } \\
\sigma\end{array}$} & $\mathrm{mm}$ & $\sigma$ & & & \\
\hline $\begin{array}{rcr}5 . & \text { und } & 6 . \\
7 . & " & 8 . \\
9 . & " & 10 . \\
11 . & " & 12 . \\
15 . & " & 16 . \\
17 . & " & 18 . \\
99 . & " & 100 . \\
101 . & \Rightarrow & 102 .\end{array}$ & $\begin{array}{l}2,50 \\
2,50 \\
2,50 \\
2,50 \\
2,60 \\
2,70 \\
2,70 \\
2,60\end{array}$ & $\begin{array}{l}2,80 \\
2,80 \\
2,80 \\
2,80 \\
2,90 \\
3,00 \\
3,00 \\
2,90\end{array}$ & $\begin{array}{l}8,75 \\
8,75 \\
8,75 \\
8,75 \\
9,10 \\
9,45 \\
9,45 \\
9,10\end{array}$ & $\begin{array}{r}9,80 \\
9,80 \\
9,80 \\
9,80 \\
10,15 \\
10.50 \\
10,50 \\
10,15\end{array}$ & $\begin{array}{l}0,3 \\
0,3 \\
0,3\end{array}$ & $\begin{array}{l}1,05 \\
1,05 \\
1,05\end{array}$ & & $\begin{array}{l}25,85 \\
26,00 \\
26,00 \\
26,55 \\
26,50 \\
26,70 \\
32,05 \\
32,30\end{array}$ & $\begin{array}{l}25,70 \\
25,90 \\
26,05 \\
26,30 \\
26,60 \\
26,70 \\
32,20 \\
32,30\end{array}$ \\
\hline $\begin{array}{ll}151 . " & 152 . \\
158 . & \\
154 .\end{array}$ & $\begin{array}{l}2,20 \\
2,25\end{array}$ & $\begin{array}{l}2,55 \\
2,60\end{array}$ & $\begin{array}{l}7,70 \\
7,88\end{array}$ & & & 1,22 & & & 95,8 \\
\hline $\begin{array}{lll}211 . & \text { " } \\
213 . & 212 . \\
215 . & 214 . \\
223 . & 216 . \\
222 . & \end{array}$ & $\begin{array}{l}2,50 \\
2,55 \\
2,55 \\
2,55\end{array}$ & $\begin{array}{l}2.90 \\
2,95 \\
2,95 \\
2,95\end{array}$ & $\begin{array}{l}8,75 \\
8,92 \\
8,92 \\
8,92\end{array}$ & $\begin{array}{l}10,1 \\
10,8 \\
10, \\
10,3\end{array}$ & $\begin{array}{l}0,4 \\
0,4 \\
0,4 \\
0,4\end{array}$ & $\begin{array}{l}1,40 \\
1,40 \\
1,40 \\
1,40\end{array}$ & & $\begin{array}{l}37,25 \\
37,30 \\
37,35 \\
37,15\end{array}$ & $\begin{array}{l}37,30 \\
37,30 \\
37,20\end{array}$ \\
\hline $\begin{array}{lll}227 . & 228 . \\
235 . & " 236 . \\
239 . & " 240 . \\
245 . & " 246 .\end{array}$ & $\begin{array}{l}2,85 \\
3,05 \\
3,00 \\
3,20\end{array}$ & $\begin{array}{l}3,15 \\
3,35 \\
3,30 \\
3,50\end{array}$ & $\begin{array}{r}9,97 \\
10,67 \\
10,50 \\
11,20\end{array}$ & $\begin{array}{l}11,02 \\
11,72 \\
11,55 \\
12,25\end{array}$ & $\begin{array}{l}0,3 \\
0,3 \\
0,3 \\
0,3\end{array}$ & $\begin{array}{l}1,05 \\
1,05 \\
1,05 \\
1,05\end{array}$ & & $\begin{array}{l}37,80 \\
36,70 \\
36,45 \\
36,00\end{array}$ & $\begin{array}{l}36,60 \\
36,45 \\
36,00\end{array}$ \\
\hline
\end{tabular}

Wie schon früher bemerkt wurde und auch aus der Tabelle ersichtlich ist, wurden auch bei diesem Versuche die ersten Kurvenpaare wegen ihrer nicht genügenden Regelmässigkeit zur Ausmessung 
nicht verwertet. Ferner lässt sich schon an der Kurventafel ohne Muhe erkennen, wie zunächst die Grösse der Latenzstadien im allgemeinen unter geringfügigen Schwankungen allmählich etwas abnimmt, was meistens recht deutlich bei jenen Versuchen zum Ausdruck kam, bei denen besonders kräftige Muskeln verwendet wurden. So war diese Abnahme bei dem zuletzt besprochenen Versuche bis zur 154. Zuckung in recht ausgeprägtem Maasse nachweisbar, während nach den darauffolgenden 50 nicht angeschriebenen Zuckungen sich die Latenzstadien der von der unteren Reizstelle ausgelösten Zuckungen wieder annäbernd gleich gross gestalteten wie zu Beginn des Versuches. Im weiteren Verlaufe desselben tritt schliesslich eine verhältnismässig ziemlich rasche Zunahme dieses Stadiums entsprechend der bereits weiter vorgeschrittenen Ermüdung des Muskels ein, welch' letztere sich vor allem an der stets stärker werdenden Dehnung der Zuckungskurven kundgibt, wobei sie durch die kurze, eingeschaltete Ruhepause nur in geringem Grade vorübergehend vermindert erscheint.

Im Anschlusse an die Besprechung dieses Versuches soll hier übrigens hervorgeboben werden, dass bei zwei anderen derartigen Versuchen, die mit einem ebenfalls ganz besonders grossen und äusserst kräftig entwickelten Tiere angestellt wurden, eine auch nur geringfügige Zunahme der Differenzwerte der Latenzstadien $\mathrm{nicht}$ nachzuweisen war, obwohl bei beiden Versuchen über 200 Zuckungen angeschrieben worden waren. Desgleichen ist hier auch daran zu erinnern, dass bei jenem Versuche, auf den sich die zuerst mitgeteilte Tabelle bezieht, die Zunahme jener Differenzgrösse erst nach mehr als 300 Zuckungen, also bedeutend später festgestellt werden konnte. Es dürften demnach wobl auch in dieser Hinsicht nicht unbedeutende, individuelle Verschiedenheiten bestehen.

Die Tatsache, dass die bei diesen Versuchen meist gefundene $\mathrm{Ab}$ nahme der Fortpflanzungsgeschwindigkeit der Nervenerregung nach einer kurzen Ruhepause wieder verschwindet, muss wohl, wie gesagt, zugunsten der Auffassung sprechen, dass es sich hier um ein wirkliches Ermüdungssymptom handle. Es ist ja klar, dass damit von vornherein die Möglichkeit ausgeschlossen erscheint, jene Abnahme als Symptom beginnenden Absterbens des Nerven zu deuten. Zur sicheren Kontrolle wurde eine Anzahl von Versuchen auch in der Art aus- 
geführt, dass nach den ersten Kurvenpaaren eine längere Pause von 5-7 Minuten eingeschaltet wurde, die zum mindesten eben so lánge dauerte wie ein gewöhnlicher Versuch ohne Unterbrechung. Hierbei zeigte sich, wie ja wohl zu erwarten war, dass eine solche Zeitdauer an und für sich keinen Einfluss auf die Grösse der Fortpflanzungsgesehwindigkeit auszuüben vermag.

Ferner muss bei diesen Überlegungen auch das Moment einer eventuell eintretenden lokalen Ermüdung (im Sinne Bernsteins) durch die länger andauernde, bzw. fortgesetzt sich wiederholende Einwirkung des elektrischen Stromes an den Reizstellen selbst mit berücksichtigt werden.

In der Tat hat $\mathrm{Nicolai^{1 }}$ ) in seinen Untersuchungen am Riechnerven des Hechtes eine "Verlängerung der Leitungszeit nach mehrfacher Reizung". beobachtet, die „im wesentlichen“, wie er feststellen konnte, „in der Nähe der Reizstelle" stattfindet und hier durch eine Schädigung derselben infolge des wiederholten elektrischen Reizes verursacht wird. Was aber die Verzögerung der Fortpflanzungsgeschwindigkeit auf der vom Reiz nicht geschädigten Nervenstrecke betrifft, so will Nicolai dieselbe nur zum Teil aus der gleichzeitigen Abuahme der Aktionsströme erklärt wissen, da "kleine Aktionsströme sich wahrscheinlich etwas langsamer fortpflauzen als grosse“. Dieser Anteil der Verzögerung sei somit analog der "geringen Verlangsamung bei Anwendung schwächerer Reize, die einen nicht 'maximalen Aktionsstrom zur Folge haben ". Weiterhin meint , er aber, „dass die ja sicher vorhandene Ermüdung doch einen merklichen Einfluss auf die Leitungsgeschwindigkeit ausübt", zu dessen Sicherstellung jedoch grössere Versuchsreihen nötig wären.

Um nun wieder auf meine Versuche zurückzukommen, so muss dazu bemerkt werden, dass hier jenes Moment der lokalen Ermüdung bzw. Schädigung durch die Induktionsschläge an den Reizstellen selbst an und für sich als Drsache für eine Zunabme der Latenzdifferenzen kaum in Betracht kommen könnte, da ja in dieser Beziehung an beiden Reizstellen dieselben Verhältnisse gegeben sind, die etwaigen Falles eine gleichmässige Verlängerung der Latenzzeiten verursachen, ihre Differenzen aber nicht verändern würden.

1) G. Fr. N i cola i, Über Ungleichförmigkeiten in der Fortpflanzungsgeschwindigkeit des Nervenprinzips, nach Untersuchangen am marklosen Riechnerven des Hechtes. Arch. f. Anat. u. Physiol., physiol. Abt. Supplbd. S. 341.1905. 
Es müsste denn höchstens sein, dass vielleicht eine lokale Ermüdung und Irritation an der unteren Reizstelle infolge der sich so oft daselbst wiederholenden elektrischen Schläge gewissermaassen ein peripheres Hindernis für die Fortleitung der an der oberen Reizstelle gesetzten Erregung abgäbe, so dass dadureh jene eine Verzögerung erfahren würde. Für diesen Fall könnte dann auch wohl die so bewirkte Verlangsamung der Leitungsgeschwindigkeit eventuell durch eine kurze Ruhepause wieder behoben werden.

Um nun darüber Aufschluss zu erlangen, wurden schliesslich die Versuche noch in folgender Weise modifiziert:

Zunächst wurde nach Tötung des Versuchstieres mit dem Nervmuskelpräparat der einen Seite ein Versuch in der Weise ausgeführt, dass eine längere Zuckungsreihe angeschrieben wurde, wobei der Nerv stets nur an einer und derselben Stelle erregt wurde, die in möglichst weiter Entfernung vom Muskel gelegen war. Sodann kam mit dem jetzt erst angefertigten Nervmuskelpräparat der anderen Seite der Parallelversuch zur Ausführung, bei dem die Nervenreizung ganz nahe am Muskel stattfand.

Auch von dieser Versuchsreihe seien hier als Beispiel einige Zahlen angeführt. $\mathrm{Zu}$ den beiden Versuchen, auf die sie sich beziehen, wurde ein mittelgrosses Tier benützt, und zwar wurde zuerst der Versuch mit hoher, d. h. zentraler Nervenreizung ausgeführt, wobei die Reizstelle $45 \mathrm{~mm}$ vom Muskel entfernt war, sodann jener , mit der peripheren Reizung des Nerven nahe am Muskel in einer Entfernung von $5 \mathrm{~mm}$. In beiden Versuchen war ferner der $\Lambda$ bstand der Durchtrennungsstelle von der Reizstelle gleich gross gewählt und betrug $10 \mathrm{~mm}$, so dass auch in dieser Beziebung beide Male analoge Verhältnisse gegeben waren. Dasselbe galt auch sowohl hinsichtlich des verwendeten Rollenabstandes $(180 \mathrm{~mm})$ als auch der Grösse der Belastung (30 g).

Dieser Doppelversuch nun ergab, wie auch die meisten anderen derartigen, in beträchtlicher Anzahl ausgeführten Versuche, dass die Latenzstadien bei zentraler Nervenreizung in stärkerem Maasse bzw. früher zunehmen als bei peripherer Lage der Reizstelle, wie dies aus der beigefügten Tabelle (S. 451) leicht zu ersehen ist.

Es ist noch hervorzuheben, dass diese Parallelversuche stets in der oben mitgeteilten Reihenfolge ausgefürt wurden, so dass also der Versuch mit peripherer Nervenreizung an jenem 
Versuche über die Ermüdbarkeit des markhaltigen Nerven.

Versuch Nr, 57 und 58 am 8. Juli 1910.

\begin{tabular}{|c|c|c|c|c|c|c|c|}
\hline \multicolumn{4}{|c|}{ Versuch Nr. 57 (zentrale Reizung) } & \multicolumn{4}{|c|}{ Versuch Nr. 58 (periphere Reizung) } \\
\hline \multirow{2}{*}{ Zuckung } & \multicolumn{2}{|c|}{$\begin{array}{c}\text { Latenz- } \\
\text { stadium als }\end{array}$} & \multirow{2}{*}{$\begin{array}{l}\text { Zuckungs- } \\
\text { höhen } \\
\text { nichtredu- } \\
\text { ziert } \\
\text { mm }\end{array}$} & \multirow{2}{*}{ Zuckung } & \multicolumn{2}{|c|}{$\begin{array}{l}\text { Latenz- } \\
\text { stadium als }\end{array}$} & \multirow{2}{*}{\begin{tabular}{|} 
Zuckungs- \\
höhen \\
nicht redu- \\
ziert \\
mm
\end{tabular}} \\
\hline & $\begin{array}{l}\text { Weg- } \\
\text { strecke mm }\end{array}$ & $\begin{array}{c}\text { Meitdaner } \\
\sigma\end{array}$ & & & $\begin{array}{c}\text { Weg- } \\
\text { strecke num }\end{array}$ & $\begin{array}{c}\text { Zeitdauer } \\
\sigma\end{array}$ & \\
\hline $\begin{array}{l}1 . \\
2 . \\
3 . \\
4 . \\
7 . \\
8 .\end{array}$ & $\begin{array}{l}2,45 \\
2,50 \\
2,45 \\
2,50 \\
2,50 \\
2,50\end{array}$ & $\begin{array}{l}8,57 \\
8,75 \\
8,57 \\
8,75 \\
8,75 \\
8,75\end{array}$ & $\begin{array}{l}14,15 \\
14,65 \\
15,30 \\
15,70 \\
16,80 \\
17,15\end{array}$ & $\begin{array}{r}1 . \\
2 . \\
3 . \\
4 . \\
7 . \\
10 .\end{array}$ & $\begin{array}{l}2,15 \\
2,20 \\
2,20 \\
2,25 \\
2,20 \\
2,20\end{array}$ & $\begin{array}{l}7,52 \\
7,70 \\
7,70 \\
7,87 \\
7,70 \\
7,70\end{array}$ & $\begin{array}{l}15,65 \\
16,15 \\
16,75 \\
17,50 \\
18,65 \\
19,40\end{array}$ \\
\hline $\begin{array}{l}171 . \\
179 . \\
180 . \\
181 . \\
182 .\end{array}$ & $\begin{array}{l}2,95 \\
3,05 \\
3,10 \\
3,05 \\
3,05 \\
\end{array}$ & $\begin{array}{l}10,32 \\
10,67 \\
10,85 \\
10,67 \\
10,67\end{array}$ & $\begin{array}{l}19,40 \\
19,20 \\
19,20 \\
19,10 \\
19,00 \\
\end{array}$ & $\begin{array}{l}172 . \\
177 . \\
180 . \\
182 . \\
184 .\end{array}$ & $\begin{array}{l}2,60 \\
2,65 \\
2,65 \\
2,65 \\
2,65 \\
\end{array}$ & $\begin{array}{l}9,10 \\
9,27 \\
9,27 \\
9,27 \\
9,27 \\
\end{array}$ & $\begin{array}{l}19,55 \\
19,25 \\
19,05 \\
18,95 \\
18,85 \\
\end{array}$ \\
\hline $\begin{array}{l}\text { Die Mittelwerte } \\
\text { der } 1 \text {. Gruppe } \\
32 \text {. }\end{array}$ & $\begin{array}{l}2,48 \\
3 ; 04 \\
\end{array}$ & $\begin{array}{r}8,69 \\
10,63 \\
\end{array}$ & $\begin{array}{l}15,625 \\
19,18 \\
\end{array}$ & $\begin{array}{c}\text { Die Mittelwerte } \\
\text { der 1. Gruppe } \\
2 \quad\end{array}$ & $\begin{array}{l}2,20 \\
2,64 \\
\end{array}$ & $\begin{array}{l}7,69 \\
9,23 \\
\end{array}$ & $\begin{array}{l}17,35 \\
19,13 \\
\end{array}$ \\
\hline $\begin{array}{c}\text { Die Grösse der } \\
\text { betreffenden } \\
\text { Zunahmen }\end{array}$ & 0,56 & 1,94 & 3,555 & $\begin{array}{l}\text { Die Grösse der } \\
\text { betreffenden } \\
\text { Zunahmen }\end{array}$ & $0, \mathbf{4 4}$ & $\mathbf{1 , 5 4}$ & 1,78 \\
\hline
\end{tabular}

Präparate stattfand, das erst einige Zeit (ungefähr eine halbe Stunde) nach erfolgter Tötung des Tieres angefertigt worden war; und dennoch ergab sich das oben angeführte Resultat entsprechend jenen in den früheren Versuchen gewonnenen Ergebnissen. Damit dürfte aber wohl auch der Einwand als nicht begründet erscheinen, dass die aus den zuerst beschriebenen Versuchen gefolgerte Abnahme der Fortpflanzungsgeschwindigkeit eventuell darauf zurückzuführen sei, dass die untere Reizstelle infolge lokaler Ermüdung durch direkte schädliche Beeinflussung von seiten des elektrischen Reizes sich allmählich gleichsam zu einem peripheren Hindernis entwickle, das seinerseits die Leitungsgeschwindigkeit verzögern würde.

Endlich sei besonders darauf hingewiesen, dass sich die Abnahmeder Fortpflanzungsgeschwindigkeit der Nervenerregung, wie sie bei diesen Versuchen allem Anscheine nach als ein Ermüdungssymptom auftritt, bereits bei einer verhältnismässig geringfügigen Inanspruchnahme des Nerven zu entwickeln scheint: der hierbei in Betracht kommenden Nervenstrecke wurden ja keine tetanischen Dauererregungen, sondern bei den zuerst besprochenen Versuchen ungefähr nur jede dritte 
452 L. Haberlandt: Versuche über die Ermüdbarkeit des markh. Nerven.

Sekunde, bei der eben beschriebenen Modifikation alle anderthalb Selzunden allerdings längere Zeit hindurch Einzelerregungen zugeführt.

Ich babe die vorliegenden Versuchsergebnisse mitgeteilt, trotzdem die Geschwindigkeit der benutzten Schreibfläche nicht eine so grosse war, wie sie für Bestimmungen der Leitungsgeschwindigkeit im markhaltigen Nerven wohl angezeigt erscheint. Ich bin mir auch vollkommen bewusst, dass die hier veröffentlichten Versuche aus diesem Grunde gewiss nicht jene weitgehende Genauigkeit besitzen, die für ganz sichere Schlussfolgerungen erforderlich ist.

Über Versuche mit tetanischen Dauerreizungen soll im Laufe der nächsten Zeit berichtet werden. 